\title{
Ratio scaling of taste intensity by a matching procedure
}

\author{
LAWRENCE E. MARKS and LINDA M. BARTOSHUK \\ John B. Pierce Foundation Laboratory and Yale University, New Haven, Connecticut 06519
}

\begin{abstract}
Given the assumptions that the taste sense is analytic and that the total perceived intensity of a complex taste equals the sum of the perceived intensities of the components, a new procedure was devised to scale taste intensity. Subjects matched the intensity of test substances either to the total intensity or to the intensity of one component of two-component mixtures (in which the two components appeared equally intense). Given the above-stated assumptions, such a pair of matches should define a psychological ratio of 2:1. Scales erected by this procedure agreed well with scales determined by magnitude estimation.
\end{abstract}

Taste sensations are commonly treated as if they are psychological compounds made up of (usually) four qualitative components. In one of the variants of Stevens' (1958) method of magnitude estimation, for example, subjects are called upon to divide up the total perceived intensity of each taste stimulus into so many units each of the components "sweet," "sour," "salty," and "bitter" (Smith \& McBurney, 1969). (Sometimes, a category "other" is added.) An implicit postulate underlies the use of this procedure: The total perceived taste intensity equals the simple sum of the perceived intensities of the four components. This postulate, which may be termed a principle of analytic addition (Marks, 1978, 1979), has several interesting implications and applications. One of these applications forms the focus of the present paper: The principle of analytic addition provides a new way to generate a psychological scale of taste magnitude.

A little background is in order. The principle of analytic addition has an analogue in psychoacoustics: The overall loudness of a complex tone (a tone with several frequency components) equals the sum of the perceived partial loudnesses of the components. Note that this principle does not deny the existence of masking, or other interactions, among tone components. Two tones in combination may partially mask one another, so that the loudness of each component is smaller than it would be if that tone were heard in isolation. What the principle does say is that the total loudness of the two-tone complex equals the sum of the two partial loudnesses. The postulate

We thank U. Jackson, who collected a portion of the data as part of a research project at Yale College. Supported by NSF Grant BNS 76-09950 to Lawrence E. Marks and NIH Grant ES-00880 to Linda M. Bartoshuk. Requests for reprints may be directed to either author at the John B. Pierce Foundation Laboratory, 290 Congress Avenue, New Haven, Connecticut 06519. of analytic addition is implicit in work as early as that of Fletcher (1940) and is found in certain current models (e.g., that of Zwicker \& Scharf, 1965) for calculating the loudness of complex sounds.

One of us (Marks, 1978) recently pointed out a noteworthy application of the principle. If the principle is correct, then it becomes a relatively simple and straightforward matter to devise an intensitymatching procedure for generating a psychophysical function. Consider a compound stimulus-say, a sound with two pure-tone components or a taste with two qualities-constructed in such a way that the two constituents are subjectively equal. It follows from the principle of analytic addition that the total subjective magnitude of the compound stimulus is twice the subjective magnitude of each of the components. Given this 2:1 ratio, it is then possible to derive a scale of sensation by a pair of matching operations: First, match the perceived magnitude of a test stimulus to the perceived magnitude of one of the constituents of the compound; next, match the perceived magnitude of the test stimulus to the total magnitude of the compound. This pair of matches should yield test-stimulus levels with subjective magnitudes in the ratio of $2: 1$. By repeating the series of matching operations at various points along the intensity continuum, one can generate a scale of sensation magnitude for the test stimulus.

Marks (1978) applied this procedure to the scaling of loudness of a $2,000-\mathrm{Hz}$ tone (the test tone), which was matched to two kinds of compound stimuli: One compound stimulus comprised combinations of pure tones of 2,000 and $5,000 \mathrm{~Hz}$ (frequencies separated far enough apart to affect each other minimally if at all); the other comprised combinations of a $2,000-\mathrm{Hz}$ tone and a narrow band of noise centered at $2,000 \mathrm{~Hz}$ [a combination that should maximize interaction (masking) between components]. The scales, derived under the assumption of analytic 

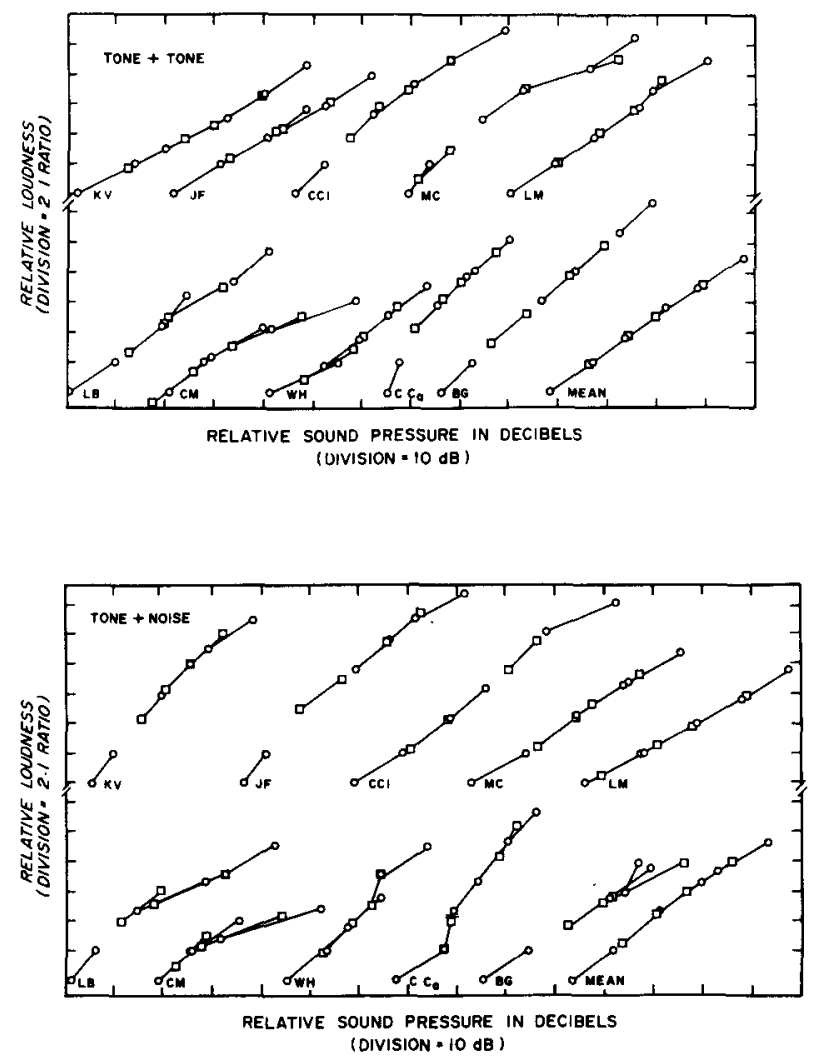

Figure 1. Scales for the loudness of a $2,000-\mathrm{Hz}$ tone, derived from a loudness-matching procedure. Each loudness ratio of 2:1 (connected pair of circles or squares) corresponds to matches of the 2,000-Hz test tones to the total loudness and to the loudness of one component of two-component combinations in which the components appear equally loud. The combinations comprised a $2,000-\mathrm{Hz}$ and a $5,000-\mathrm{Hz}$ tone (upper figure) or a $2,000-\mathrm{Hz}$ tone and a narrow band of noise centered on $2,000 \mathrm{~Hz}$ (lower figure). Results are plotted both individually for the 10 subjects and pooled across subjects. (From Marks, 1978).

addition, were virtually identical under the two conditions, despite the difference in degree of masking within the mixtures. Figure 1 shows both the average results and the results for the 10 individual subjects. The scales approximate power functions, and, in fact, the average scales come close to Stevens' (1955) sone scale. The average scales are proportional to the .68 power of sound pressure, while the sone scale is proportional to the .60 power.

There are (at least) two ways to interpret this outcome. (1) If one accepts the validity of the sone scale of loudness, as derived from numerical judgment procedures like magnitude estimation, then the data shown in Figure 1 may be taken as evidence for the validity of the principle of analytic addition in certain two-component sounds. (2) Alternately, if one accepts the principle of analytic addition, then the data may be taken as evidence for the validity of the numerical judgments of loudness that provide the basis for the sone scale.

The purpose of the present study was to determine whether scaling taste intensity on the basis of analytic addition yields a similar outcome. Scales of taste intensity were derived by having subjects mix pairs of substances with relatively pure taste qualities $[\mathrm{HCl}$, sour; sucrose, sweet; quinine hydrochloride $(\mathrm{QHCl})$, bitter] to subjective equality, and then match single substances to the overall intensity and to the component intensities of the mixtures. For comparison, the same subjects also made magnitude estimations of the perceived intensities of concentrations of the matching stimuli; this makes it possible to compare the scales obtained by matching, as based on the principle of analytic addition, to the scales obtained by direct quantitative judgment. Psychophysical functions for taste vary both with the substance tasted and also with the way the taste solutions are presented (flowed passively over the tongue or sipped into the mouth and then expectorated). Accordingly, we varied both test substances and presentation procedures in order to assure that a range of psychophysical functions would result. This permits a strong test of the scaling model.

\section{METHOD}

\section{Subjects}

Subjects came from a pool of 26 men and women, including seven staff members and 19 student volunteers. The students were paid to participate. Most of the subjects had had previous experience in making psychophysical judgments and in taste experiments. Ten subjects from the pool served in each of the four experimental conditions.

\section{Apparatus and Stimulus Presentation}

Stimuli. Sucrose (reagent grade) and demineralized water were used to construct $.1-, .2-$, and .4-M solutions. Two-component mixtures were constructed by adding small volumes of high concentrations of the desired second component (i.e., QHCl, Baker grade; $\mathrm{HCl}$, reagent grade).

Flow. The dorsal flow method consists of flowing solutions across the extended tongue at a rate of $4 \mathrm{mI} \mathrm{sec}^{-1}$ (McBurney \& Pfaffmann, 1963). Each taste stimulus was preceded by a 20 -sec water rinse, and both rinse and taste stimuli were warmed to $34^{\circ} \mathrm{C}$, approximately the temperature of the extended tongue.

Sip and spit. With this method, subjects sip from cups containing $10 \mathrm{ml}$ of solution. The tasting of each stimulus was preceded by a thorough water rinse, and both rinse and taste stimuli were at room temperature, approximately $22^{\circ} \mathrm{C}$.

\section{Test Conditions}

There were four main conditions: (A) scaling bitter $(\mathrm{QHCl})$, flow presentation; (B) scaling sweet (sucrose), flow presentation; (C) scaling sweet (sucrose), sip and spit; (D) scaling sour $(\mathrm{HCl})$, sip and spit. The substances and modes of presentation were selected in order to produce a variety of psychophysical functions. In each condition, taste intensity was scaled both by magnitude estimation and by intensity matching as described below.

\section{Procedure}

The subjects first made magnitude estimations of the taste magnitude of a series of concentrations of the substance under investigation in that condition. For example, in Condition $\mathbf{A}$ 
Table 1

Molar Concentrations Used for Magnitude Estimation of Taste Intensity

\begin{tabular}{lccc}
\hline Condition A & Condition B & Condition C & Condition D \\
\hline .0000100 & .100 & .071 & .0005 \\
.0000185 & .141 & .100 & .000793 \\
.0000342 & .200 & .141 & .00126 \\
.0000633 & .282 & .200 & .00199 \\
.000117 & .398 & .282 & .00316 \\
.000216 & .562 & .398 & .0079 \\
.00074 & .794 & .562 & .0050 \\
.00137 & 1.122 & .794 & \\
.00253 & & 1.122 & \\
\hline
\end{tabular}

Note-Condition $A=Q H C l$ (flow), Condition $B=$ sucrose (flow), Condition $C=$ sucrose (sip), Condition $\mathrm{D}=\mathrm{HCl}$ (sip).

(bitter, flow), 10 concentrations of $\mathrm{QHCl}$ were presented in random order, and the subject's task was to assign numbers in proportion to the intensity of the taste experience. The range of concentrations was preselected so as to be sure to include the range scaled by matching in the next part of the experiment. Each concentration level was presented and judged twice in the course of this initial part of the experiment (three times each in Conditions B, C, and D). Tabie 1 lists the concentrations used in each of the four conditions.

After a 5-min rest came the scaling by matching. The matching procedure had two parts: (1) construction of a two-component mixture with constituents equally strong perceptually; and (2) matching the substance to be scaled to one constituent and then to total taste intensity. Again, let us use the scaling of bitter (Condition A) as an example. First, the subject tasted the initial standard concentration of sucrose (.1 M), to which a small amount of $\mathrm{QHCl}$ had been added. The subject judged whether the sweet component or the bitter component was the stronger. If the sweet, more $\mathrm{QHCl}$ was added on the next trial; if the bitter, less $\mathrm{QHCl}$ was added. By means of a bracketing procedure, the concentration of $\mathrm{QHCl}$ was adjusted until a solution was obtained that the subject judged equally sweet and bitter. This $\mathrm{QHCl} /$ sucrose mixture became the standard mixture for the scaling by matching.

The standard mixture was presented and the subject was instructed to attend to the bitter component. Then a level of unmixed $\mathrm{QHCl}$ was presented and the subject judged whether its intensity was greater or less than the intensity of the bitter component of the standard mixture. If greater, the concentration of QHCI was decreased on the next trial; if less, the concentration was next increased. The up-down procedure was continued until four reversals of judgment occurred. (In a few instances, the first reversal deviated markedly from the next three, so the first reversal was discarded and a fifth added.) The standard mixture was available whenever the subject requested it.

Following the matching to one component, the standard mixture was again presented, and the subject was instructed to attend to its total taste intensity. Then the matching procedure was repeated just as before, with the subject now judging whether the intensity of unmixed $\mathrm{QHCl}$ was greater or less than the total intensity of the mixture.

The entire sequence-construction of the mixture, matching to one component, matching to total intensity-was then repeated twice, each time starting the construction of the mixture with a higher concentration of the fixed component sucrose $(.2 \mathrm{M}$, then $.4 \mathrm{M}$ ). Thus the experimental procedure made it possible to determine pairs of matches at each of three regions along the concentration continuum. These same three concentrations of sucrose were used as fixed components of the mixtures in all four conditions (A, B, C, and D) of the experiment.

\section{RESULTS AND DISCUSSION}

Figure 2 gives the results of both the magnitudeestimation scaling and of the scaling-by-matching, pooled over the 10 subjects who participated in each condition. Filled circles given geometric means of the magnitude estimates. Open circles and open squares give scale values inferred from the taste matches, as determined by the following procedure: Recall that in each condition, the subject produced pairs of matches at three places along the intensity continuum-at each place, the subject matched the magnitude of a test substance to the total magnitude and to the magnitude of one component of a taste mixture. These matching concentrations were averaged geometrically across subjects, producing-for each condition and each place along the continuum-a pair of mean matching concentrations that, according to the postulate of analytic addition, should have a subjective ratio of 2:1. In each section of Figure 2, the lower pair of circles gives the matches made to one component and to the total at the lowest of the three levels; the points are plotted so that the psychological scale values have a ratio of $1: 2$. The pairs of squares give the corresponding average matches at the medium level of concentration (the points adjusted in the vertical plane so that the lower square falls on the line segment that connects the first pair of circles), and the upper pairs of circles give the matches at the highest level of concentration (adjusted in the vertical plane so the lower point falls on the line connecting

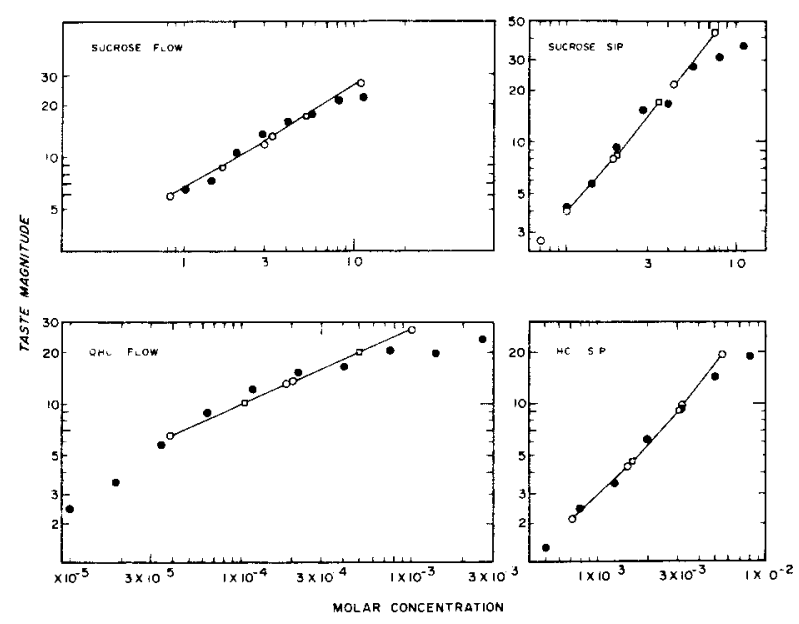

Figure 2. Scales of taste intensity. Filled circles are geometric means of magnitude estimates. Open circles and squares are scale values derived from a taste-intensity matching procedure. Each taste ratio of 2:1 (connected pair of circles or squares) corresponds to matches of the test substance to the total intensity and to the intensity of one component of two-component mixtures in which the components appear equally intense. 
the squares); again, the scale values assigned to each pair are in a ratio of $1: 2$.

The overall agreement between the two types of scaling is remarkable. In the double logarithmic coordinates of Figure 2, the sip-and-spit stimulus presentations (sucrose, sip; $\mathrm{HCl}$, sip) gave relatively steep psychophysical functions, whereas the flow presentation (sucrose, flow; $\mathrm{QHCl}$, flow) gave flatter functions, the quinine function being the flattest of all, and this was true for scales obtained both by magnitude estimation and by matching. The scales obtained by matching approximate power functions (straight lines); indeed, these scales approximate power functions better than do the scales obtained by magnitude estimation. All of the magnitude-estimation scales show some bending over at the highest concentrations. Still, what is most notable about the results is the agreement between the two sets of scales - the way the scales determined by the two scaling procedures vary together when the substance scaled and the mode of presentation are changed.

This is not to deny that there are some differences between the scales obtained by the two procedures. As already noted, the magnitude-estimation functions in Figure 2 show some deceleration, while the functions obtained by matching are straighter (or perhaps show a bit of acceleration). Moreover, if one ignores the curvature in the magnitude-estimation data and fits straight lines to them nonetheless, in each case the magnitude-estimation function is slightly flatter than the corresponding matching function: That is, the magnitude estimates systematically yield slightly smaller power-function exponents.

Table 2 lists the power-function exponents determined for the 10 individual subjects in each condition. In calculating each individual exponent for magnitude estimation, we excluded those extremely low (and high) concentrations that fell more than one concentration step below (or above) the lowest (or highest) concentration that the subject set in the matching experiment. For example, in the lower lefthand panel of Figure 2 (quinine, flow), one can see that the range of concentrations for magnitude estimation extends well beyond the range of concentrations set in matching. For such a set of data, the magnitude estimates for the two lowest and the one highest concentration would be excluded in calculating the exponent. This insures that the magnitudeestimation and matching exponents are calculated over the same portion of the stimulus range.

As Table 2 shows, the differences in exponent between magnitude estimation and matching amount to some 10\%-25\%. Perhaps this outcome should not surprise, for the matching task is a type of "production" procedure, and Stevens often noted that psychophysical functions obtained by magnitude

Table 2

Power-Function Exponents from Magnitude Estimation (ME) and from Matching (M)

\begin{tabular}{|c|c|c|c|c|c|c|c|c|}
\hline \multirow[b]{2}{*}{ Subject } & \multicolumn{2}{|c|}{ QHCl (Flow) } & \multicolumn{2}{|c|}{ Sucrose (Flow) } & \multicolumn{2}{|c|}{ Sucrose (Sip) } & \multicolumn{2}{|c|}{$\mathrm{HCl}(\mathrm{Sip})$} \\
\hline & $\mathbf{M E}$ & $\mathbf{M}$ & $\mathrm{ME}$ & $\mathbf{M}$ & ME & $\mathbf{M}$ & ME & $\mathbf{M}$ \\
\hline 1 & .392 & .491 & .274 & .841 & .983 & 1.163 & .815 & 1.160 \\
\hline 2 & .520 & .418 & 1.105 & .648 & & & 1.151 & .939 \\
\hline 3 & .341 & .388 & & & & & & \\
\hline 4 & .620 & .843 & .823 & .539 & 1.174 & 1.024 & .808 & 1.206 \\
\hline 5 & .265 & .275 & & & & & & \\
\hline 6 & .450 & .471 & & & & & & \\
\hline 7 & .473 & .485 & .269 & .669 & 1.396 & 1.243 & & \\
\hline 8 & .020 & .559 & .471 & .495 & & & & \\
\hline 9 & .337 & .414 & & & & & & \\
\hline 10 & .380 & .564 & & & & & & \\
\hline 11 & & & .246 & .634 & & & & \\
\hline 12 & & & .769 & .891 & & & & \\
\hline 13 & & & .464 & .401 & & & .761 & 1.361 \\
\hline 14 & & & .687 & .507 & & & & \\
\hline 15 & & & .439 & .566 & & & & \\
\hline 16 & & & & & .771 & .720 & .685 & 1.171 \\
\hline 17 & & & & & 1.638 & 1.237 & 1.511 & 1.211 \\
\hline 18 & & & & & 1.400 & 2.012 & & \\
\hline 19 & & & & & .848 & 1.202 & & \\
\hline 20 & & & & & .763 & 1.696 & & \\
\hline 21 & & & & & .957 & .985 & & \\
\hline 22 & & & & & .412 & .956 & & \\
\hline 23 & & & & & & & .883 & .874 \\
\hline 24 & & & & & & & 1.203 & 1.531 \\
\hline 25 & & & & & & & .738 & 1.194 \\
\hline 26 & & & & & & & .875 & 1.207 \\
\hline Mean & .440 & .491 & .555 & .619 & 1.034 & 1.224 & .943 & 1.185 \\
\hline SD & .114 & .142 & .269 & .145 & .350 & .357 & .249 & .176 \\
\hline
\end{tabular}


estimation typically have lower exponents than corresponding functions obtained by magnitude production (e.g., Stevens \& Greenbaum, 1966), and he argued that some average between estimation and production gives the "best" estimate of the exponent. Meiselman, Bose, and Nykvist (1972) found that production gave larger exponents than estimation in scaling taste intensity. It is likely that magnitude production would have yielded functions with exponents at least as great as the values obtained by the present matching procedure.

It is worth noting the ranges of the individual exponents listed in Table 2, particularly that the exponents derived from matching show considerable variability, though not quite as much, on the average, as do the exponents from magnitude estimation. Similar variation in exponents from matching appears in Marks' (1978) analogous data on loudness. While a large measure of the individual variation in magnitude-estimation exponents may readily be attributed to differences in the ways people use numbers, variation in matching exponents is not so readily accounted for. Recall that the size of the matching exponent is inversely related to the ratio of concentrations matched to two aspects of the taste mixtures. While some of this variation in taste matching may represent "noise," some of it may reflect real individual differences in sensory functioning. And, by the model of analytic addition, such sensory-based differences in matching imply corresponding individual differences in the underlying psychophysical functions for taste. It is premature to speculate at this point on the possible nature or extent of such individual differences; we would like to suggest, however, that it is in just such a realm as individual differences in taste functioning that the usefulness (which may ultimately be the same as the validity) of scaling by matching will show itselfin helping to further our understanding of how sensory systems operate.

In conclusion, the present study of taste intensity, like Marks' (1978) earlier study of loudness, shows good agreement between magnitude scales generated by operations of matching and magnitude scales determined by direct estimation. The scales generated by matching were derived on the premise that the taste sense shows analytic addition of intensity across quality. McBurney and Gent (1979) recently reviewed evidence on the question of whether the taste sense is analytic or synthetic, and concluded that the evidence leans toward taste being analytic. If the principle of analytic addition-upon which is based the scaling by matching - is taken as given, the results suggest that numerical estimation procedures have at least first-order validity. Alternatively, if one accepts as valid the numerical estimations, the results support the validity of the principle of analytic addition in the sense of taste.

\section{REFERENCES}

Fletcher, H. Auditory patterns. Reviews of Modern Physics, $1940,12,47-65$.

MARKS, L. E. PHONION: Translation and annotations concerning loudness scales and the processing of auditory intensity. In N. J. Castellan, Jr., \& F. Restle (Eds.), Cognitive theory (Vol. 3). Hillsdale, N.J: Erlbaum, 1978.

MARKS, L. E. A theory of loudness and loudness judgments. Psychological Review, 1979, 86, 256-285.

McBurney, D. H., \& Gent, J. F. On the nature of taste qualities. Psychological Bulletin, 1979, 86, 151-167.

McBurney, D. H., \& Pfaffmann, C. Gustatory adaptation to saliva and sodium chloride. Journal of Experimental Psychology, $1963,65,523-529$.

Meiselman, H. L., Bose, H. E., \& Nykvist, W. F. Magnitude production and magnitude estimation of taste intensity. Perception \& Psychophysics, 1972, 12, 249-252.

Smith, D. V., \& McBurneY, D. H. Gustatory cross-adaptation: Does a single mechanism code the salty taste? Journal of Experimental Psychology, 1969, 80, 101-105.

Stevens, S. S. The measurement of loudness. Journal of the Acoustical Society of America, 1955, 27, 815-829.

Stevens, S. S. Problems and methods of psychophysics. Psychological Bulletin, 1958, 55, 177-196.

Stevens, S. S., \& Greenbaum, H. B. Regression effect in psychophysical judgment. Perception \& Psychophysics, 1966, 1, 439-446.

Zwicker, E., \& Scharf, B. A model of loudness summation. Psychological Review, 1965, 72, 3-26.

(Received for publication May 17, 1979; revision accepted August 9, 1979.) 